\title{
Asociación de la obesidad abdominal y alteraciones de la presión arterial con estrategias de afrontamiento ante la carestía alimentaria en niños en edad escolar
}

Peraza-López Enrique Eduardo ${ }^{1}$, Zapata-Peraza Alicia Leonor ${ }^{2}$

${ }^{1}$ Facultad de Medicina, Universidad Autónoma de Yucatán. ${ }^{2}$ Centro de Investigaciones Regionales “Dr. Hideyo Noguchi, Universidad Autónoma de Yucatán

\section{ABSTRACT}

Food shortages and abdominal obesity in schoolchildren. An association study.

Introduction. Cardiovascular diseases and the complications of diabetes mellitus account for most of adult mortality in Mexico. Abdominal obesity (AO) and alterations in blood pressure (BP) are two of its fundamental determinants. The pathological changes caused by these entities have been demonstrated in children from 3 years of age.

Objective. To analyze the association between socioeconomic status, episodes of food shortages, sedentary lifestyle and soft drinks consumption with the development of obesity and alterations in BP in children of school age in an elementary school during 2015-2016 in Mérida Yucatán México.

Materials and methods. 264 participants, aged between 6 and 12 years were evaluated for weight, height, abdominal perimeter, BP. Semi-structured interviews were used to determine socioeconomic status, episodes of food shortages, soft drinks consumption, and sedentary lifestyle. Descriptive statistics were determined; Chi-square tests and Student's T-tests were used for association analysis.

Results. A prevalence for overweigh in terms of BMI of $28.4 \%$ was found, $28 \%$ for abdominal obesity, and $15.7 \%$ for alterations of BP. Abdominal obesity and BP alterations were associated with consumption of bottled soft drinks $(p<0.001)$. In case of food shortages, the coping strategy (loan vs reorganization was asociated with $\mathrm{AO}(\mathrm{p}=0.019)$. The sex of the participant was not associated with BMI, although it was with $\mathrm{AO}(\mathrm{p}<0.01)$.

Conclusions. Socioeconomic status is associated with alterations in BMI. in those participants who had periodic shortages of food, the strategy employed for dealing with the issue determines the outcomes in terms of anthropometric variables.

\section{RESUMEN}

Introducción. Las enfermedades cardiovasculares y las complicaciones de la diabetes mellitus representan las principales causas de mortalidad en adultos en
Historial del artículo Recibido: 26 abr 2018

Aceptado: 15 ago 2018

Disponible online: 1 sep 2018

Palabras clave

Obesidad, escolares, niños, estatus socioeconómico, carestía alimentaria, hipertensión.

\section{Keywords}

Obesity, schoolchildren, children, socioeconomic status, food shortage, hypertension.

Copyright (C) 2018 por autores y Revista Biomédica.

Está trabajo esta licenciado bajo las atribuciones de la Creative Commons (CC BY).

http://creativecommons.org/licenses/by/4.0/

\footnotetext{
*Autor para correspondencia:

Enrique Eduardo Peraza López, Facultad de Medicina, UADY

correo electrónico: E_Peraza_L@hotmail.com htpp://revistabiomedica.mx.
} 
México. La obesidad abdominal (OA) y las alteraciones en la tensión arterial (TA) son dos de sus determinantes fundamentales. Las alteraciones ocasionadas por estas entidades se han demostrado en niños a partir de los tres años de edad.

Objetivo. Analizar la asociación entre el estatus socioeconómico, episodios de carestía alimentaria, sedentarismo y consumo de refrescos embotellados con el desarrollo de obesidad y alteraciones en la TA en niños en edad escolar.

Material y métodos. En 264 participantes en edad escolar (6-12 años) se determinaron peso, talla, perímetro abdominal y TA. Mediante entrevista semiestructurada se valoró estatus socioeconómico, episodios de carestía alimentaria, ingesta de refrescos embotellados y sedentarismo. Se aplicaron pruebas de Chi-cuadrada, $t$ de Student para búsqueda de asociación.

Resultados. Se encontró una prevalencia del 28.4\% para sobrepeso/obesidad en términos del IMC, 28\% para obesidad abdominal, $15.7 \%$ en alteraciones de la TA. La OA y alteraciones en TA se asociaron con consumo de refrescos embotellados $(p<0.001)$. En caso de presentarse carestía alimentaria la estrategia de afrontamiento (préstamo vs reorganización) guardó asociación significativa con OA $(p=0.019)$. El sexo del participante no se asoció con IMC, aunque si con OA $(p<0.01)$.

Conclusiones. El estatus socioeconómico guarda asociación con las alteraciones en el IMC en aquellos participantes con carestía alimentaria periódica. Las estrategias empleadas por la familia determinan en gran medida los desenlaces en términos de variables antropométricas.

\section{INTRODUCCION}

Las enfermedades crónico degenerativas se han convertido en la primera causa de morbimortalidad a nivel mundial.(1-4) De acuerdo con el Instituto Nacional de Estadística y geografía (INEGI), las cinco principales causas de mortalidad en México son, en orden decreciente: cardiopatía isquémica, complicaciones de la diabetes mellitus, neoplasias malignas, enfermedades cerebrovasculares y enfermedades hepáticas. $(5,6)$

Las enfermedades antes citadas tienen un sustrato etiológico o son agravadas por un conjunto de alteraciones metabólicas y bioquímicas que en conjunto reciben el nombre de síndrome metabólico.(1)
El síndrome metabólico es una entidad clínica constituida por un agregado de factores metabólicos, clínicos y bioquímicos los cuales incrementan en forma sinérgica el riesgo de desarrollar diversas patologías crónicas en el organismo. Las enfermedades que se han asociado con el síndrome metabólico incluyen pero no se limitan a: enfermedades cardiovasculares, diabetes mellitus tipo 2, diversos tipos de neoplasias, demencia de diversas etiologías, entre otras.(7)

Las alteraciones metabólicas implicadas en la etiología de este síndrome son sumamente diversas destacando: la resistencia a la insulina, adiposidad central (con predominio del componente visceral), dislipidemia aterogénica, disfunción endotelial, susceptibilidad genética, elevación de las cifras de tensión arterial, un estado sistémico de hipercoagulabilidad, hipercortisolismo, activación inapropiada del sistema nervioso simpático, así como un fenotipo proinflamatorio sistémico mantenido en forma crónica. $(4,7,8)$

Muchas de estas variables no son fácilmente medibles en la práctica clínica, de modo que se han generado diversas definiciones operativas con el objeto de permitir el diagnóstico con base en mediciones antropométricas y de laboratorio fácilmente disponibles en la práctica habitual. Entre las definiciones más aceptadas en la actualidad se encuentran: la definición de la OMS para el síndrome metabólico, los criterios descritos en el tercer reporte del Adult Treatment Panel (ATP-III) y la definición de síndrome metabólico por la Federación Internacional de la Diabetes (IDF, por sus siglas en inglés).(1-4,8)

Entre las variables clínicas incluidas en las definiciones antes citadas del síndrome metabólico destacan: alteraciones en el metabolismo de la glucosa, dislipidemia aterogénica, alteraciones de la presión arterial y obesidad. $(1,4,7,9)$

Entre las variables antropométricas, la obesidad juega el papel más destacado en la sospecha y definición clínica del síndrome metabólico, tanto la obesidad en términos del índice de masa corporal (IMC), como del perímetro abdominal se han relacionado con desenlaces metabólicos adversos. Particularmente esta última es requerida como 
un criterio indispensable para el diagnóstico del síndrome metabólico según la definición de la IDF. $(1,3,4)$

En relación con la etiología del síndrome metabólico, la resistencia a la acción de la insulina representa el eje central de la génesis y vinculación de las diversas condiciones patológicas comprendidas en esta entidad. Para su cuantificación se dispone de metodologías tales como la determinación del índice de resistencia a la insulina, sin embargo, la aplicación de estos instrumentos aún no se encuentra consolidada en la práctica clínica habitual. Como sucedáneo a estas mediciones se emplean alteraciones antropométricas tales como la obesidad abdominal y las bioquímicas como son la glucosa alterada en ayuno. $(4,7)$

La prevalencia mundial del síndrome metabólico no es conocida con certeza, sin embargo, algunas estimaciones, empleando los criterios de la IDF, la sitúan en al menos un $25 \%$ de la población adulta. Cabe mencionar que la distribución mundial de esta patología es heterogénea, con predominio en países desarrollados. Por ejemplo, en Estados Unidos tomando como base los criterios ATP-III, se reportó en 2003 una prevalencia del $32.9 \%$ en la población mayor de 20 años, cifra que se incrementó a $34.7 \%$ en 2012. Resulta destacable mencionar que en estos estudios resulta posible establecer el diagnóstico de síndrome metabólico en el $46.7 \%$ de los participantes con una edad de 60 años o más. (10)

La prevalencia del síndrome metabólico en México se ha inferido con base en los resultados de las Encuestas Nacionales de Salud y Nutrición (ENSANUT 2000, 2006 y 2012). Según ENSANUT 2000 , se encontró una prevalencia del $26.6 \%$ en la población con edades comprendidas entre los $20 \mathrm{y}$ 69 años, según los criterios de la IDF. En los datos proporcionados por ENSANUT 2006, la prevalencia del síndrome en mayores de 20 años se ubica en el 49.8\%.(11) En ENSANUT 2012 el 32.4\% de los adultos mayores de 20 años presentaban obesidad definida en términos de IMC, si se considera la prevalencia de sobrepeso y obesidad en forma combinada se obtiene una prevalencia del 71.6\% para este año.(12) Dicha prevalencia se ha mantenido constante, tal y como lo demuestran los resultados en la ENSANUT de Medio Camino 2016, donde se encontró una prevalencia combinada del $72.5 \%$ de sobrepeso y obesidad por IMC en mayores de 20 años. La prevalencia global de obesidad abdominal es de $76.6 \%$ y por sexo se obtiene una prevalencia del $87.7 \%$ en mujeres y del $65.4 \%$ en hombres. (13)

En Yucatán, según ENSANUT 2012, la prevalencia de sobrepeso y obesidad en los adultos fue del $82 \%$ para mujeres y $78.6 \%$ para hombres. Si se define obesidad en términos del IMC esta se encuentra presente en el $46.2 \%$ de las mujeres y el $43 \%$ de los hombres mayores de 18 años. La obesidad definida en función del perímetro abdominal se reporta en el $76 \%$ de la población yucateca(12).

Existe una carencia relativa de información respecto al comportamiento de las variables del síndrome metabólico en la población pediátrica. Ya se ha demostrado mediante estudios clínicos y anatomopatológicos que los correlatos del síndrome metabólico son identificables en edades tan tempranas como los tres años $(8,14,15)$. Una de las situaciones que dificulta el adecuado estudio del síndrome metabólico en los niños es la ausencia de un estándar de diagnóstico universalmente aceptado. Entre las guías disponibles actualmente se encuentran modificaciones de las recomendaciones del ATP-III y de la IDF, sin embargo el éxito que han registrado en la práctica clínica ha sido variable. $(4,8)$. La prevalencia de obesidad en niños y jóvenes es un problema creciente de salud pública, en el caso particular de Yucatán ENSANUT 2012 reporta en niños en edad escolar, una prevalencia de sobrepeso del 22.4\% y de obesidad del 22.7\%.(12)

Diversos estudios asocian el desarrollo de la obesidad y diversos componentes del síndrome metabólico a una dieta abundante en grasas y azúcares simples. Aunque tradicionalmente se ha asociado la obesidad con la "abundancia" propia de las naciones desarrolladas, en los países en vías de desarrollo se ha presentado un marcado incremento de estas patologías en la población de los estratos socioeconómicos inferiores, situación debida probablemente a características socioculturales y 
al acceso generalizado que actualmente poseen los alimentos procesados. $(12,16)$

\section{MATERIAL Y MÉTODOS}

Se realizó una investigación transversal, prospectiva, analítica y observacional, cuyo objetivo fue el de investigar la posible asociación entre el estatus socioeconómico familiar y la exposición a carestía alimentaria intermitente con el desarrollo de obesidad (IMC y abdominal) y alteraciones en la presión arterial en niños en edad escolar, de una escuela primaria de la ciudad de Mérida, Yucatán; en el ciclo escolar 2015-2016. El universo se encontró conformado por todos los niños en edad escolar de la escuela primaria pública Pedro R. Pinzón Sánchez de la colonia San José Tecoh, ubicada en el sur de la ciudad de Mérida, Yucatán. El tamaño muestral fue calculado mediante la fórmula para poblaciones finitas:

$$
n=\frac{N \times z_{\alpha}^{2} \times p \times q}{d^{2} \times(N-1)+z_{\alpha}^{2} \times p \times q}
$$

Dónde: $\mathbf{n}=$ tamaño de la muestra requerido
$\mathrm{N}=$ total de población que conforma el universo
$\mathrm{Z} \alpha=$ Seguridad deseada, en este caso
$95 \%$, lo cual equivale a 1.96 en tabla $\mathrm{Z}$
$\mathbf{p}=$ prevalencia estimada de la alteraciones
compatibles con obesidad abdominal en la
población estudiada $(0.50)$
$\mathrm{q}=$ complemento de $\mathbf{p}(1-\mathrm{p})$
$\mathrm{d}=$ precisión $5 \%$ (valor estándar de 0,05$)$

Con una población de 350 , se consideró como representativa una muestra de al menos 183 estudiantes. Los criterios de inclusión considerados fueron el de encontrarse cursando estudios como alumno regular en la primaria seleccionada $\mathrm{y}$ tener una edad comprendida entre 6 y 12 años. Se excluyeron aquellos estudiantes que padeciesen alguna condición médica preexistente conocida $\mathrm{o}$ que se hubiesen negado a participar en el estudio. Se eliminaron a quienes no completaron los cuestionarios o las mediciones requeridas. A cada uno se le realizaron las siguientes determinaciones antropométricas: peso $(\mathrm{Kg})$, talla $(\mathrm{cm})$, perímetro abdominal (cm), presión arterial (TA, mmHg, brazalete pediátrico). Los índices antropométricos fueron clasificados con base en los criterios para edad pediátrica de la IDF y de la National High Blood Pressure Education Program $(4,8)$.

Se recabó información concerniente a ciertas variables socioeconómicas y del estilo de vida, de los participantes, mediante una entrevista semiestructurada, las cuales fueron: actividad física del participante, consumo de alimentos industrializados y refrescos embotellados, nivel de seguridad alimentaria en el último mes, nivel socioeconómico familiar y hacinamiento doméstico.

La clasificación del participante en función de su actividad física se realizó mediante el Cuestionario Internacional de Actividad Física (IPAQ)(17), el estatus socioeconómico y la presencia de hacinamiento se determinó empleando el cuestionario 10x6 de la Asociación Mexicana de Agencias de Inteligencia de Mercado y Opinión (AMAI), empleado por INEGI, (13) el consumo de alimentos industrializados y la inseguridad alimentaria fueron evaluados mediante entrevista semiestructurada aplicada en momentos diferentes, tanto a los estudiantes como a sus representantes legales.

Los datos se procesaron con el paquete estadístico SPSS versión 20. Se determinaron los estadísticos descriptivos y se aplicaron las pruebas t de Student y Chi cuadrada para evaluar asociación. La evaluación de asociación entre las variables categóricas se realizó mediante el cálculo del Odds Ratio y del intervalo de confianza al 95\%. Durante el presente estudio se consideraron todos los principios éticos requeridos para esta clase de investigación. Se informó y solicitó el consentimiento informado por escrito a cada binomio participante-representante legal, haciendo hincapié en su capacidad de rechazar el participar en el estudio y/o suspender su participación en caso de considerarlo oportuno.

\section{RESULTADOS}

El universo estuvo conformado por la matricula completa de la escuela primaria evaluada. Previa plática informativa a padres y maestros se invitó a 
un total de 347 estudiantes del primero al sexto año de primaria a participar en el estudio. Se recopiló en forma satisfactoria la información correspondiente a 264 binomios participante-representante legal (76\% del universo). Se eliminaron del estudio los datos de 83 participantes debido a la existencia de cuestionarios incompletos. Se eliminó a un participante debido a que su edad no se encontraba dentro del rango establecido para el estudio.

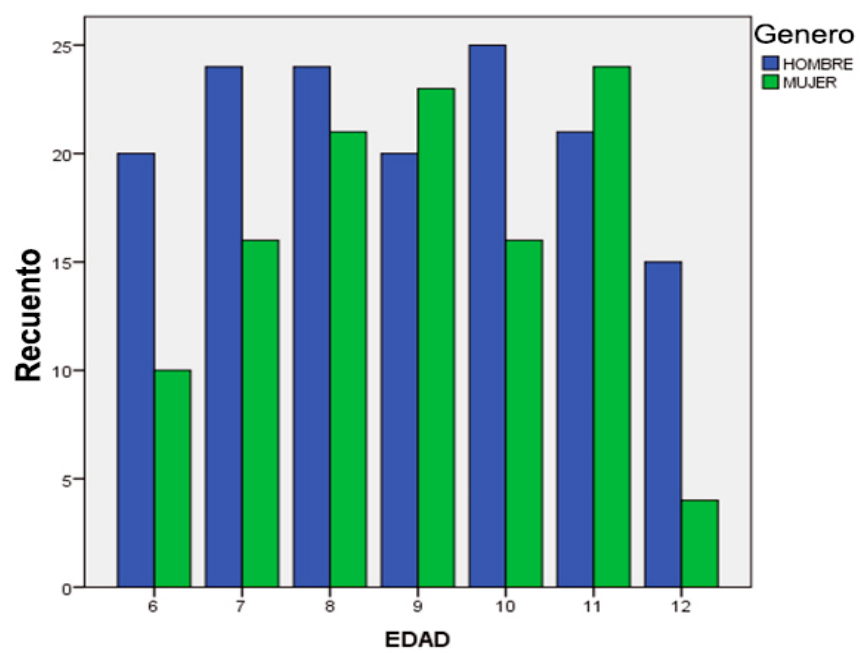

Figura 1. Distribución de los participantes por edad y sexo.

Fuente: encuesta

De los de los 263 participantes, $55.6 \%$ (149) corresponden al sexo masculino y $43.3 \%$ (114) al femenino. En la figura 1, se muestra la distribución de los participantes de acuerdo al la edad y sexo. La media de edad fue de 8.9 años (DE \pm 1.8 años), uniformemente distribuidos en número entre el primero y sexto grados de primaria, correspondiendo del $12 \%$ al $17 \%$, del total de participantes, a cada grado escolar. En lo referente al estatus socioeconómico según criterios AMAI, $71 \%$ de los participantes provienen de familias de nivel socioeconómico bajo y $29 \%$ de familias pertenecientes a clase media.

Con relación al estado nutricional de los participantes evaluado mediante IMC, 3\% (8) presentaron una cifra inferior al percentil 3 para edad y sexo, calificando como desnutrición clínica, 68.1\% (179) presentaron IMC dentro de la normalidad y $28.4 \%$ (76) presentaron un
IMC superior a percentil 85, clasificando como sobrepeso/obesidad. Se encontró una asociación estadísticamente significativa entre la edad y la prevalencia de sobrepeso/obesidad $(p<0.05)$. La presencia de sobrepeso/obesidad presentó una asociación estadísticamente significativa con el consumo habitual de refrescos embotellados (desde una porción al día) $(\mathrm{p}<0.0001$, OR 2.92, IC95\% 1.54.34). No se encontró asociación estadísticamente significativa entre la clasificación por IMC con el sexo del participante, estatus socioeconómico o condiciones de hacinamiento.

En cuanto a la alteración en el perímetro abdominal, se encontró una prevalencia del 28\% (75) de obesidad abdominal (percentil \pm 80 para edad, sexo y talla). El desarrollo de obesidad abdominal guardó asociación significativa con el sexo del participante, predominando el masculino $(\mathrm{p}=0.01$, OR 5.8 IC95\% 1.08-31.1). También hubo asociación estadísticamente significativa entre la presencia de obesidad abdominal y el consumo de refrescos embotellados ( $>0.01$ OR 1.43, IC95\%1.1-1.8), figura 2; y entre la presencia de obesidad abdominal y el vivir en condiciones de hacinamiento $(p=0.043)$. No se encontró asociación con el nivel socioeconómico de la familia.

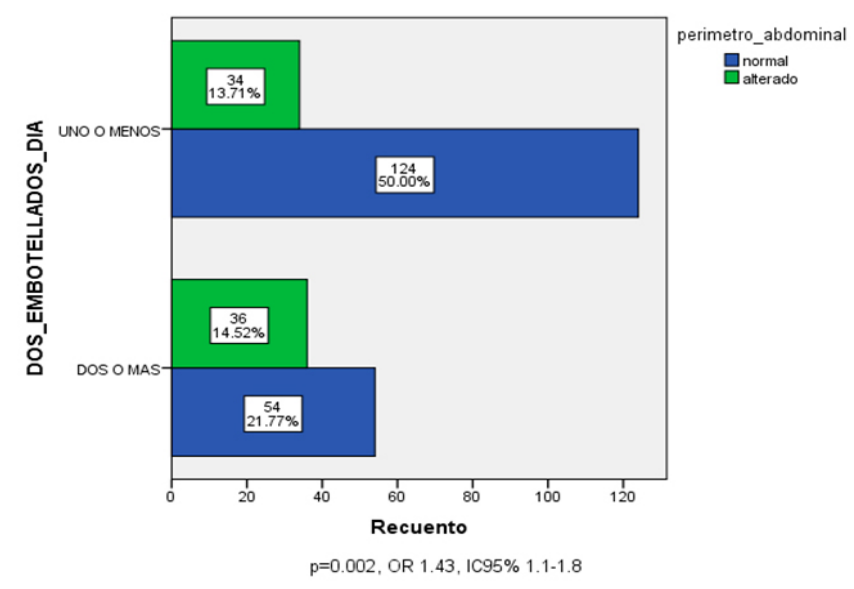

Figura 2. Relación entre el consumo de refrescos embotellados y presencia de obesidad abdominal.

Fuente: encuesta y somatometría

Se encontró una prevalencia del 15.7\% de alteraciones en la presión arterial, definida como TA en o por encima del percentil 90 en al menos 
dos de tres determinaciones. Entre éstos el 80.9\% presentó alteraciones únicamente en el componente sistólico, 9.5\% en el componente diastólico y $9.5 \%$ en ambos. La presencia de obesidad abdominal, así como la definida por IMC, presentaron asociación significativa con la presencia de alteraciones en la presión arterial, tanto en sus componentes sistólico como diastólico ( $\mathrm{p}<0.01$ OR 5.6 IC95\% 2.8-11.3).

En participantes con IMC y perímetro abdominal normal, no se encontró asociación estadística entre las alteraciones de la TA con el sexo del participante, el consumo de refrescos embotellados, inseguridad alimentaria, estatus socioeconómico ni con actividad física.

En lo referente al patrón alimentario, 53.4\% reporta consumir al menos dos porciones de alimentos procesados (chatarra) al día. En los que reportan dificultades económicas, el tipo de estrategia empleada para solventarlas se asocia estadísticamente con el patrón de ingesta de comida procesada. Aquellas familias que solventan las dificultades económicas mediante préstamos monetarios reportaron incrementar el consumo de alimentos procesados en los periodos de carestía $(\mathrm{p}<0.0001)$, por el contrario, las que basan su estrategia en la optimización de recursos, preparando alimentos en casa o las que reciben aportes en especie de otros familiares, presentan una reducción en el consumo de alimentos procesados en épocas de dificultad ( $\mathrm{p}<0.01$ IC95\% 0.048-0.08).

La existencia de períodos de carestía alimentaria se asoció en forma estadísticamente significativa con el desarrollo de alteraciones en el IMC y perímetro abdominal. En el caso de los estudiantes que reportaron haber padecido carencias alimentarias en el último mes, el empleo de préstamos económicos por parte de la familia se asoció con el desarrollo de sobrepeso/obesidad $(p<0.05)$, por el contrario, la reducción de la ingesta o calidad de los alimentos en caso de dificultades económicas se asoció en forma significativa con desnutrición $(\mathrm{p}<0.005)$.

Los participantes que reportaron algún grado de inseguridad alimentaria (35\%) presentaron una tendencia a emplear una dieta más rica en alimentos procesados que aquellos que no reportaron dificultades económicas $(\mathrm{p}=0.019)$. La presencia de inseguridad alimentaria se asocia a vivir en condiciones de hacinamiento $(p<0.01$ IC95\% 1.3-5.3). El tipo de inseguridad alimentaria (reducción de la ingesta vs suspensión de la misma), guarda relación con el sexo del participante, los del masculino reportan una frecuencia mayor de reducción en la calidad y/o cantidad de la ingesta alimentaria, sin embargo, los del femeninos refieren una frecuencia mayor de suspender por completo la ingesta durante una o más comidas en tiempos de carestía ( $\mathrm{p}=0.029$ IC95\%,1.01-4.1).

La actividad física global de los participantes ubica a la mayoría de estos como sedentarios; de acuerdo con los datos proporcionados únicamente $18 \%$ se pueden considerar como activos, mientras que el $45 \%$ de la muestra puede ser clasificada directamente como sedentaria. El pertenecer a clase social media se asoció en forma estadísticamente significativa con el sedentarismo, respecto a los participantes de clase socioeconómica baja $(\mathrm{p}=0.018)$. El consumo de alimentos procesados se asoció en forma estadísticamente significativa con una disminución del gasto energético mediado por ejercicio físico ( $\mathrm{p}=0.018$ IC95\%0.28-0.89).

El consumo de refrescos embotellados representa una práctica habitual para la mayoría de los participantes en este estudio. Únicamente $29 \%$ de los participantes afirma no consumirlos en forma regular, un $34.8 \%$ refiere consumir una porción al día y $36 \%$ refiere consumir en forma regular al menos dos porciones por día. El consumo de refrescos embotellados fue la variable que presentó una mayor asociación con el desarrollo de sobrepeso/obesidad, tanto por IMC como por perímetro abdominal $(p<0.0001$ OR 4, IC95\% 2.5-9.17).

\section{DISCUSIÓN}

El presente estudio permite tener una visión general de la diversidad de asociaciones que se presentan entre el estatus socioeconómico y el estado nutricional en niños en edad escolar en una zona económicamente desfavorecida. La muestra presentó una distribución homogénea por edad y 
sexo, condición que permite que los resultados sean comparables con los datos provenientes de las principales encuestas nacionales (ENSANUT 2000, 2006 y 2012).(12,13)

La prevalencia conjunta de sobrepeso/obesidad en el presente estudio fue del $28.4 \%$, inferior a la media nacional del $32 \%$ reportada por ENSANUT 2012 para niños en edad escolar. En Yucatán, ENSANUT 2012 reporta una prevalencia combinada de sobrepeso/obesidad en escolares del $45 \%$. Considerando únicamente obesidad medida por IMC, encontramos que la prevalencia de esta última en los participantes fue del $21 \%$, lo cual coincide con la media para la población yucateca reportada por ENSANUT 2012.(12)

Los participantes de este estudio presentaron alteraciones en la presión arterial en un $15.7 \%$, esta prevalencia resulta consistente con los hallazgos reportados en la literatura.(8). El sobrepeso/obesidad, tanto en términos del IMC como en perímetro abdominal fue la variable que presentó la asociación más significativa con el desarrollo de alteraciones en la presión arterial. Un IMC por encima de percentil 80 para edad y sexo se asocia estadísticamente con una TA por encima del percentil 90 para edad y sexo ( $>>0.001$ IC95\%2.3-9.8).

En forma similar a lo que se reporta Lurbe E (2010), no se demostró una asociación entre las alteraciones de la presión arterial y el sexo del participante, probablemente a consecuencia de que la mayor parte de las diferencias en la patología cardiovascular guardan estrecha relación con la presencia de los esteroides sexuales, mismos que sólo se encontrarán en cantidades significativas hasta después de la pubertad.(18)

Los participantes del sexo masculino presentaron una mayor prevalencia de obesidad abdominal que las del femenino, a pesar de no encontrarse diferencias significativas con relación al IMC, $(\mathrm{p}=0.01$, OR 5.8 IC95\% 1.08-31.1), lo cual coincide con lo reportado en la literatura. Debido a la influencia de las hormonas sexuales esta diferencia en la distribución de la grasa corporal es característica en la etapa postpuberal. Una explicación que se ha propuesto para este fenómeno, es la diferencia en el consumo de alimentos hipercalóricos (particularmente jarabe de maíz de alta fructosa) los cuales pueden inducir el desarrollo de resistencia a la insulina desde etapas tempranas de la vida. $(4,7,15,19)$

Acorde con lo anteriormente descrito se encontró que la obesidad abdominal se encuentra asociada con el consumo habitual de alimentos procesados ( $p<0.05$ OR 2.2 IC95\% 1.04-4.6), así como el de refrescos embotellados ( $<<0.01$ OR 1.43, IC95\%1.11.8).

En el presente estudio, se encontró una asociación estadísticamente significativa entre una ingesta de grandes cantidades de energía y el desarrollo de sobrepeso/obesidad, los participantes que reportan incrementar su consumo de alimentos procesados, durante los periodos de carestía alimentaria, poseen una tendencia mayor al desarrollo de alteraciones del IMC con relación a sus contrapartes que no reportan inseguridad alimentaria.(20)

Los resultados de prevalencia de sobrepeso y obesidad encontrados en este estudio resultan comparables a los obtenidos en otras investigaciones efectuadas en la localidad. En 2010, Sierra encontró una prevalencia de sobrepeso y obesidad del 12.1 y $21.3 \%$ respectivamente; (21), en otro estudio efectuado en una unidad hospitalaria en Mérida, Yucatán en el mismo año por Nahuat E. se determinó una prevalencia combinada de sobrepeso/ obesidad del 33.1\%. A pesar de que las poblaciones son esencialmente diferentes, las prevalencias encontradas resultan prácticamente idénticas.(22)

En 2010, Mora S. demuestra la presencia de alteraciones aterogénicas en los lípidos séricos en el 71\% de una cohorte de niños obesos en Mérida, Yucatán. A pesar de que en éste no fue posible evaluar la presencia de dislipidemia, la elevada prevalencia de obesidad abdominal sugiere la presencia probable de patologías concomitantes. Debido a lo anterior, se considera pertinente realizar nuevas investigaciones que evalúen en forma integral los diversos factores que constituyen el síndrome metabólico en la población pediátrica en la entidad. (23)

Uno de los hallazgos más destacados, en la presente investigación, corresponde a la asociación encontrada entre las dificultades para 
la adquisición de alimentos y el desarrollo de sobrepeso/obesidad, fue así que la relación que se presentó entre la carestía alimentaria con las variaciones en el IMC, dependieron del tipo de estrategia empleada para solventarla. De tal forma que las familias que recurrieron a préstamos económicos en época de dificultad presentaron una tendencia a incrementar el consumo de alimentos procesados durante la carestía. Por el contrario, aquellas familias que respondieron reorientando su alimentación hacia el consumo de alimentos preparados en casa, basándose en el apoyo, en especie, por parte de familiares presentan una marcada disminución en las alteraciones en IMC/ perímetro abdominal ( $p=0.02$ IC95\% 1.14-4). Lo anterior, puede explicarse en parte con base en los mecanismos para el manejo del estrés ocasionado por la inestabilidad económica. Resulta probable que aquellas familias que emplean préstamos económicos como estrategia para superar la carestía alimentaria presenten niveles de estrés superiores a aquellas acostumbradas a responder a las dificultades mediante reorganización de sus patrones de comportamiento (estrategias de afrontamiento pasivas vs proactivas). Se ha descrito en la literatura que bajo condiciones de estrés, las personas tienden a la búsqueda de satisfactores inmediatos como un paliativo para el malestar emocional que sufren en ese momento.

Consideramos que este enfoque en particular resulta interesante para ser profundizado en estudios posteriores. En caso de confirmarse estos hallazgos, el desarrollo de habilidades de manejo de la economía familiar, con énfasis en la optimización de los recursos y una adecuada regulación emocional podrían resultar en una estrategia efectiva para la prevención del sobrepeso y obesidad en este subgrupo de pacientes.(20)

Entre las limitantes del presente estudio, destaca el que éste se realizó en una sola escuela primaria pública en una zona urbana. Las asociaciones encontradas pueden no resultar directamente extrapolables a otras circunstancias, particularmente en el medio rural (incluso en medio urbano en situación económica más favorecida).
Una fuente potencial de sesgo en la presente investigación se debió a que únicamente fueron incluidos los datos de aquellos participantes a los que resultó posible realizar la entrevista semiestructurada tanto a ellos como a sus representantes legales. A pesar de que se incluyó en el estudio al $76 \%$ de la matricula estudiantil, es posible que el $24 \%$ restante difiera significativamente del resto de la muestra, en particular en lo referente al involucramiento de los representantes en las actividades escolares, así como en su estabilidad laboral.

\section{CONCLUSIONES}

El sobrepeso y la obesidad representan un problema de salud pública identificable desde etapas tempranas de la vida. El estatus socioeconómico, la carestía alimentaria y los métodos para el afrontamiento de la misma son algunas de las situaciones que determinan el patrón de ingesta y gasto calórico en niños en edad escolar.

En el desarrollo del sobrepeso/obesidad y alteraciones de la tensión arterial, el consumo de alimentos procesados y refrescos embotellados juega un papel central. Dado que el patrón de afrontamiento de la carestía alimentaria en aquellas familias que se encuentran expuestas a la misma, guarda asociación con los desenlaces en relación al sobrepeso/obesidad, resulta necesario profundizar en las características particulares, que inducen a cada familia a reaccionar de uno u otro modo ante esta clase de dificultad. En caso de confirmarse ésta, el desarrollo de intervenciones encaminadas al desarrollo de estrategias conductuales y de autorregulación, en los integrantes de aquellas familias expuestas a carestía alimentaria, podría resultar particularmente efectivo para disminuir la prevalencia de sobrepeso/obesidad en éstos.

\section{REFERENCIAS}

1. Third Report of the National Cholesterol Education Program (NCEP) Expert Panel on Detection, Evaluation, and Treatment of High Blood Cholesterol in Adults (Adult Treatment Panel III) Final Report. Circulation 2002 Dec 17;106(25):3143.

2. Marchesini G, Forlani G, Cerrelli F, Manini R, Natale $\mathrm{S}$, Baraldi L, et al. WHO and ATPIII proposals for the 
definition of the metabolic syndrome in patients with Type 2 diabetes. Diabet Med J Br Diabet Assoc 2004 Apr;21(4):383-7.

3. Zerati AE, Monteiro Guimarães AL, Miranda de Carvalho HA, Saes GF, Ragazzo L, Wolosker N, et al. Influence of criteria used in determining prevalence of metabolic syndrome (NCEP-ATPIII versus IDF) in patients with intermittent claudication. Ann Vasc Surg 2014 Apr;28(3):640-3.

4. Alberti KGMM, Zimmet P, Shaw J. Metabolic syndrome - a new world-wide definition. A consensus statement from the international diabetes federation. Diabet Med 2006;23(5):469-480.

5. Durán-Arenas L, Ávila-Palomares PD, ZendejasVillanueva R, Vargas-Ruiz MM, Tirado-Gómez LL, López-Cervantes M. Costos directos de la hemodiálisis en unidades públicas y privadas. Sal Públ Méx 2011;53: 516-524.

6. Instituto Nacional de Estadística y Geografía. Mujeres y hombres en México 2016 [Internet]. INEGI; 2016. Available from: http://internet.contenidos.inegi.org.mx/ contenidos/Productos/prod_serv/contenidos/espanol/ bvinegi/productos/nueva estruc/702825084097.pdf

7. Kaur J. A Comprehensive Review on Metabolic Syndrome. Cardiol Res Pract. 2014;2014:1-21.

8. The Fourth Report on the Diagnosis, Evaluation, and Treatment of High Blood Pressure in Children and Adolescents. Pediatrics 2004 Aug 1;114(Supplement 2):555.

9. Jellinger PS, Handelsman Y, Rosenblit PD, Bloomgarden ZT, Fonseca VA, Garber AJ, et al. American Association Of Clinical Endocrinologists And American College Of Endocrinology Guidelines For Management Of Dyslipidemia And Prevention Of Cardiovascular Disease. Endocr Pract 2017 Apr;23(Supplement 2):1-87.

10. Aguilar M, Bhuket T, Torres S, Liu B, Wong RJ. Prevalence of the metabolic syndrome in the United States, 2003-2012. Jama 2015;313(19):1973-74.

11. Rojas R, Aguilar-Salinas CA, Jiménez-Corona A, Shamah-Levy T, Rauda J, Ávila-Burgos L, et al. Metabolic syndrome in mexican adults: results from the National Health and Nutrition Survey 2006. Sal Públ Méx 2010;52:S11-S18.

12. Oropeza Abúndez C, Instituto Nacional de Salud Pública (Mexico), Mexico, editors. Encuesta nacional de salud y nutrición 2012: resultados nacionales. Primera edición. Cuernavaca, Morelos, México: Instituto Nacional de Salud Pública : Secretaría de Salud; 2012. 195 p.

13. Hernandez M, Rivera J, Shamah T, Cuevas L, Gomez L, Gaona E, et al. Encuesta Nacional de Salud y Nutricion de Medio Camino 2016 (Ensanut 2016). Informe final de resultados [Internet]. México: Instituto Nacional de Salud Pública: Secretaría de Salud; 2016. 1-149 p. Available from: http://promocion.salud.gob.mx/dgps/ descargas1/doctos 2016/ensanut mc 2016-310oct.pdf

14. Pacifico L, Chiesa C, Anania C, De Merulis A, Osborn $\mathrm{JF}$, Romaggioli S, et al. Nonalcoholic fatty liver disease and the heart in children and adolescents. World $\mathrm{J}$ Gastroenterol WJG. 2014 Jul 21;20(27):9055-71.

15. Alterio A, Alisi A, Liccardo D, Nobili V. Non-Alcoholic Fatty Liver and Metabolic Syndrome in Children: A Vicious Circle. Horm Res Paediatr 2014;82(5):283-9.

16. Coca-colonization of diets in the Yucatan. - PubMed NCBI [Internet]. [cited 2018 Feb 27]. Available from: https://www.ncbi.nlm.nih.gov/pubmed/15950095

17. Mantilla Toloza SC, Gómez-Conesa A. El Cuestionario Internacional de Actividad Física. Un instrumento adecuado en el seguimiento de la actividad física poblacional. Rev Iberoam Fisioter Kinesiol:48-52.

18. Lurbe E, Cifkova R, Cruickshank JK, Dillon MJ, Ferreira I, Invitti C, et al. Manejo de la hipertensión arterial en niños y adolescentes: recomendaciones de la Sociedad Europea de Hipertensión. Hipertens Riesgo Vasc 2018;35(1):47-74.

19. Mirmiran P, Yuzbashian E, Asghari G, HosseinpourNiazi S, Azizi F. Consumption of sugar sweetened beverage is associated with incidence of metabolic syndrome in Tehranian children and adolescents. Nutr Metab 2015;12:25.

20. Unikel Santoncini C, Díaz de León Vázquez C, Márquez $\mathrm{R}$, Alberto J. Conductas alimentarias de riesgo y correlatos psicosociales en estudiantes universitarios de primer ingreso con sobrepeso y obesidad. Sal Ment 2016 Jun;39(3):141-8.

21. Sierra F. Prevalencia de desnutrición, sobrepeso y obesidad en niños de 1 a 12 años de edad que asisten a los comedores infantiles del DIF municipal (Mérida) [Tesis de Licenciatura en Nutricion]. [México]: Universidad Autonoma de Yucatán; 2010.

22. Nahuat E. Estado de nutrición y su relación con la morbilidad en niños de 5 a 14 años atendidos en el servicio de pediatria del Hospital [Tesis de Licenciatura en Nutricion]. [México]: Universidad Autonoma de Yucatán; 2010.

23. Mora $\mathrm{S}$. Correlación entre los índices que miden obesidad (IMC o CC) con los lípidos totales plasmáticos, en niños entre 6 y 15 años con diagnóstico de obesiad en una unidad de medicina familiar. [Tesis recepcional de la Especialidad en Medicina Familiar]. [México]: Universidad Autonoma de Yucatán; 2010. 